\title{
Maternal heart rate patterns under resting conditions in late pregnancy
}

\author{
Hein Odendaal ${ }^{1 *}$, Coen Groenewald ${ }^{1}$, Michael MMyers ${ }^{2,3,45}$ and William P Fifer ${ }^{2,3,4,5}$ \\ ${ }^{1}$ Department of Obstetrics and Gynaecology, Faculty of Medicine and Health Sciences, Stellenbosch University, Cape Town, Western Cape, South Africa \\ ${ }^{2}$ Department of Psychiatry, Columbia University Medical Center, New York, NY, USA \\ ${ }^{3}$ Department of Pediatrics, Columbia University Medical Center, New York, NY, USA \\ ${ }^{4}$ Division of Developmental Neuroscience, New York State Psychiatric Institute, New York, NY, USA \\ ${ }^{5}$ Extraordinary Professor, Department of Obstetrics and Gynaecology, Faculty of Medicine and Health Sciences, Stellenbosch University, Cape Town, Western \\ Cape, South Africa
}

\begin{abstract}
Objectives: To describe maternal heart rate patterns observed during antenatal monitoring under resting conditions between the gestational ages of 34 to 38 weeks and to demonstrate its associations with uterine activity.

Methods: Each participant had five high quality ECG electrodes attached to her anterior abdominal wall which were connected to the Monica AN24 device to collect raw electrical signals from the maternal and fetal ECG and signals of uterine activity. Proprietary software was then used to download the raw data and extract the maternal and fetal heart rate patterns and uterine activity.
\end{abstract}

Results: Several distinct maternal heart rate patterns were observed. These included unusually high or low levels of variability, tachycardia, bradycardia, regular and irregular periodic changes and sporadic changes where the heart rate suddenly decreased or increased. Some of the fluctuations, especially decelerations of maternal heart rate, seemed to be associated with uterine activity.

Conclusion: The clinical implications of these different patterns, for both the mother and fetus, needs to be explored further. There is a need for computerized analyses of the different maternal patterns during different gestational ages to determine its relevance.

Synopsis

Various maternal heart rate patterns under resting conditions in late pregnancy are described.

\section{Introduction}

Poor maternal cardiac function is associated with reduced fetal growth as reflected by reduced birth weight and preterm delivery, with reduced cardiac output and maternal cyanosis suggested as underlying mechanisms [1]. Importantly, cardiac disease is becoming one of the leading cause of maternal death in developed countries because of increased maternal age and extended survival of patients with congenital heart disease [2]. As heart rate is one of the determinants of cardiac output [3], it is essential to know more about the significance of ranges of maternal heart rates in pregnancy. Another developing field of interest is assessment of heart rate variability (HRV) as a biomarker for autonomic nervous system (ANS) function [4].

There is a paucity of literature regarding maternal heart rate (MHR) under resting conditions during pregnancy, before the onset of labour. There is also little information about the periodic effects of uterine activity on the MHR prior to the onset of labour. Reports on incidental monitoring of the MHR by scalp electrodes [5,6] or ultrasound transducers provide little information on different MHR patterns $[7,8]$.

The Monica AN24 ${ }^{\mathrm{TM}}$ (Monica Health Care, Nottingham, UK) uses the raw data obtained from five electrodes placed on the anterior abdominal wall of the mother. The fetal (f) ECG signal quality is significantly better during the first stage of labor in comparison to Doppler cardiotocography and the monitor can provide a continuous printout of the MHR with less MHR/FHR ambiguity when compared to the cardiotocograph [9]. In addition, it records electrical impulses from the uterus to illustrate contraction patterns resembling what is observed with direct recordings of intrauterine pressure [10].

More information on the MHR is emerging, as part of the data collected in the Safe Passage Study, where fetal heart rate patterns and fetal movements were recorded at different gestational ages [11]. The transabdominal recording of the fetal heart rate was feasible as early as 20-24 weeks gestation [12]. Here we describe several unique patterns which have appeared in this data set and demonstrate its association with uterine activity at 34 to 38 weeks gestation.

${ }^{*}$ Correspondenceto: Hein Odendaal, Department of Obstetrics and Gynaecology, Faculty of Medicine and Health Sciences, Stellenbosch University, Cape Town, Western Cape, South Africa, Tel. + 2721938 9601/9221, E-mail: hjo@sun.ac.za

Key words: maternal heart rate, patterns, pregnancy, variability

Received: August 25, 2018; Accepted: September 10, 2018; Published: September 12, 2018 


\section{Methods}

The Safe Passage Study was designed to investigate the role of prenatal alcohol exposure (PAE) in 12,000 pregnancies. Recruitment included low and high risk pregnancies, with a wide range of exposures to alcohol, nicotine, cannabis and methamphetamine. Stillbirth was one of the primary outcomes. Participants from the clinical site in South Africa were recruited at a local community health centre. All assessments were done at Tygerberg Hospital. All participants had an ultrasound examination to confirm the gestational age. Ethical approval was obtained from the Health Research Ethics Committee of Stellenbosch University (Ethics approval number: N06/10/210).

Recordings were carried out in quiet rooms between $08 \mathrm{~h} 00$ and $16 \mathrm{~h} 00$ on weekdays with participants lying at a $15^{\circ}$ right or left lateral tilt. For the late gestation recordings (34-38 weeks GA), four electrodes were placed on the anterior maternal abdomen, one $5 \mathrm{~cm}$ above the umbilicus, one just above the pubic hairline, and the other two laterally, with one $10 \mathrm{~cm}$ to the right and the other $5 \mathrm{~cm}$ to the left of the umbilicus. The fifth electrode was placed $5 \mathrm{~cm}$ lateral to the electrode on the right side. Before application, the skin was lightly abraded to remove superficial dry squamous cells which could reduce electrode impedance. The skin was washed with soap and water if the participant had recently used skin ointment. After application, the electrodes were connected to the Monica AN24 monitoring device which was attached to the abdominal wall with an elastic band to prevent it from moving and to ensure a similar position across all of the studies. The recordings lasted at least 50 minutes under resting conditions. During recordings, tracings of the fetal or maternal heart rate could not be seen by the operator or participant. Fetal and maternal heart rates and a tracing reflecting uterine contraction were extracted from the raw data using the DK 2.2 software provided by Monica. For this study examples of different MHR patterns were selected from recordings in more than 5,000 participants.

\section{Results}

We observed several different MHR patterns such as decreased or increased variability, tachycardia, bradycardia, decreased or increased baseline variability, regular and irregular periodic changes and sporadic changes where the heart rate suddenly decreased or increased. No participant had cardiovascular pathology.

Figure 1 illustrates a MHR which varied around $70 \mathrm{bpm}$. At the beginning of the recording the MHR rapidly increased to above 100 bpm but returned quickly to the baseline. Towards the end of the recording the MHR rapidly increased to $90 \mathrm{bpm}$, then decreased but did not return to the basal rate. Figure 2 illustrates a case where the MHR varied from around $60 \mathrm{bpm}$ to just above $70 \mathrm{bpm}$ on a few occasions with no periodic changes.

As illustrated in Figure 3, there were cases where the MHR fluctuated rapidly between 110 and $140 \mathrm{bpm}$ making it difficult to detect a baseline. In some cases there seemed to be an association between the MHR changes and uterine activity (Figure 4) while in other cases the association with uterine contractions was less clear (Figure 5). In Figure 6 the MHR was noted to fluctuate between 65 and $135 \mathrm{bpm}$. Here the association with uterine activity was less clear but the rapid decline in MHR followed periods of greater uterine activity (Figure 6). In other cases it was observed that the basal MHR fluctuated around $115 \mathrm{bpm}$

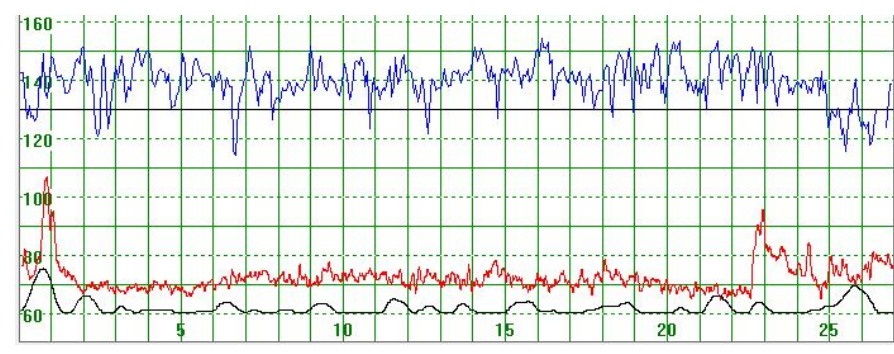

Figure 1. The upper part of the graph depicts a normal fetal heart rate. The middle part illustrates the maternal heart rate (MHR) which varies around $70 \mathrm{bpm}$. At the beginning of the recording the MHR rapidly increased to above $100 \mathrm{bpm}$ but returned rapidly to the baseline. Towards the end of the recording the MHR rapidly increased to $90 \mathrm{bpm}$ but then decreased but not to the basal rate. The lower graph gives an indication of uterine activity The vertical scale gives the heart rate in beats per minute and the horizontal scale time in minutes.

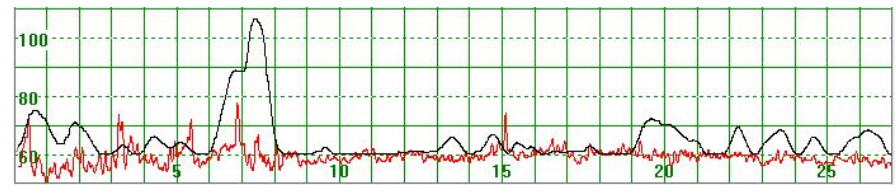

Figure 2. The MHR varies around $60 \mathrm{bpm}$

The vertical scale gives the heart rate in beats per minute and the horizontal scale time in minutes

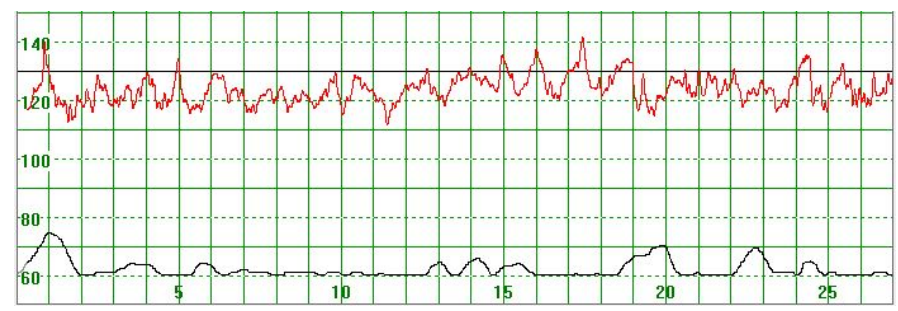

Figure 3. The MHR varies around $120 \mathrm{bpm}$. No periodic changes are observed The vertical scale gives the heart rate in beats per minute and the horizontal scale time in minutes.

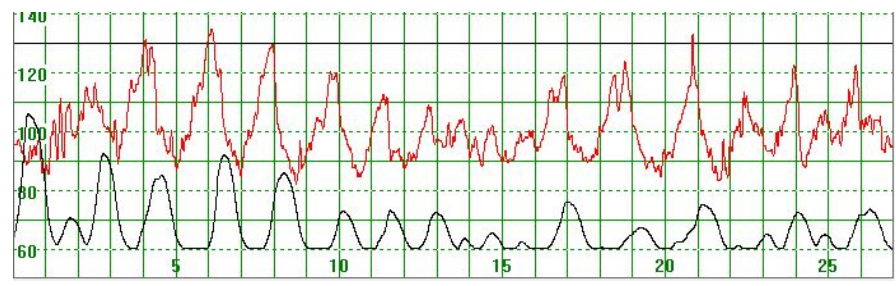

Figure 4. The MHR fluctuates rapidly between 90 and $130 \mathrm{bpm}$. It is difficult to detect the baseline. There seems to be an association between the MHR changes and uterine activity The vertical scale gives the heart rate in beats per minute and the horizontal scale time in minutes.

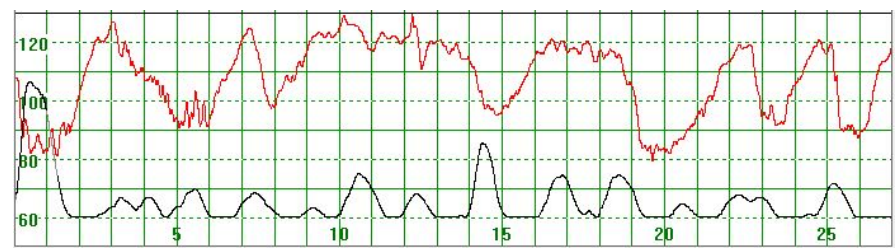

Figure 5. The MHR fluctuates between 90 and $130 \mathrm{bpm}$. The association with uterine contractions is less clear. The basal heart rate seems to be around $120 \mathrm{bpm}$

The vertical scale gives the heart rate in beats per minute and the horizontal scale time in minutes. 
but was preceded and followed by periods of a lower rate $(80 \mathrm{bpm})$ where the association with uterine activity was less clear (Figure 7). Figure 8 shows a different pattern where the initial MHR varied around $120 \mathrm{bpm}$ but then declined rapidly to much lower levels when uterine activity decreased (Figure 8).

Overall the success rate in abstracting the MHR from the 8 cases assessed varied from $97.7 \%$ to $99.6 \%$. The mean MHR ranged from 60.9 to $123.3 \mathrm{bpm}$ (Table 1). Short term variability ranged from 5.2 to 32.7 $\mathrm{ms}$ and the mean minute range from 43.3 to $147.0 \mathrm{~ms}$.

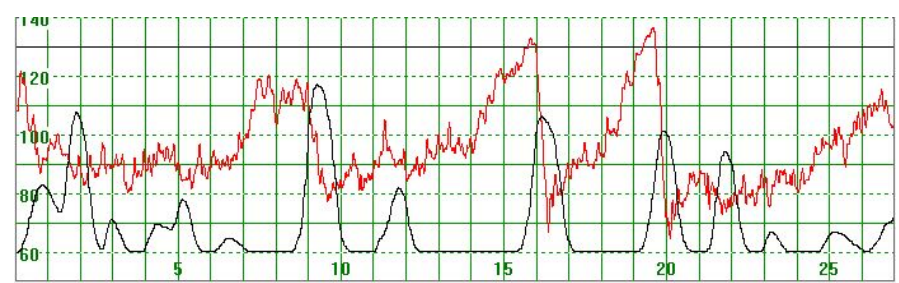

Figure 6. The MHR fluctuates between 65 and $135 \mathrm{bpm}$. Association with uterine activity is less clear but a rapid decline in MHR seems to follow periods of greater uterine activity The vertical scale gives the heart rate in beats per minute and the horizontal scale time in minutes.

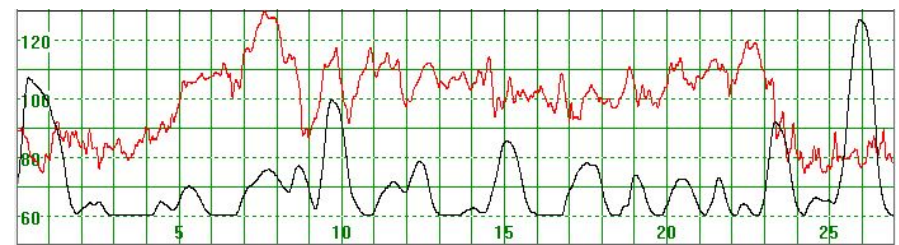

Figure 7. The basal MHR fluctuates around $115 \mathrm{bpm}$ but is preceded and followed by periods of a lower. Association with uterine activity is less clear

The vertical scale gives the heart rate in beats per minute and the horizontal scale time in minutes.

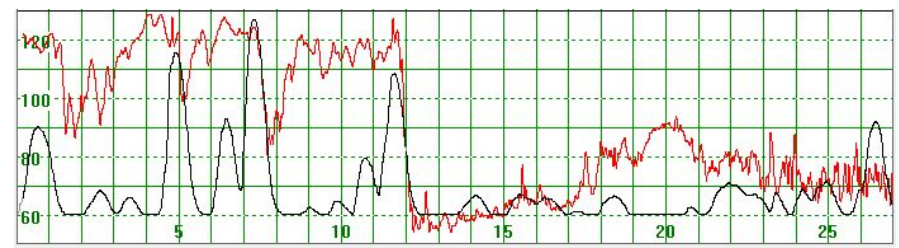

Figure 8. The initial MHR varies around $120 \mathrm{bpm}$ but then declines rapidly to much lower levels when uterine activity also became less

The vertical scale gives the heart rate in beats per minute and the horizontal scale time in minutes.

Table 1. Analyses of maternal heart rate by software of the system

\begin{tabular}{|c|c|c|c|c|}
\hline Participant & $\begin{array}{c}\text { Success Rate } \\
(\mathbf{\%})\end{array}$ & $\begin{array}{c}\text { Mean MHR } \\
\mathbf{( b p m )}\end{array}$ & $\mathbf{S T V}^{\mathbf{b}} \mathbf{( m s )}$ & $\mathbf{M M R}^{\mathbf{c}} \mathbf{( m s )}$ \\
\hline 1 & 99.5 & 73.6 & 21.5 & 109.6 \\
\hline 2 & 98.4 & 60.9 & 32.7 & 147.0 \\
\hline 3 & 97.7 & 123.3 & 7.8 & 43.3 \\
\hline 4 & 99.6 & 100.5 & 16.8 & 123.1 \\
\hline 5 & 99.5 & 104.7 & 5.2 & 61.6 \\
\hline 6 & 99.1 & 96.6 & 13.7 & 101.5 \\
\hline 7 & 99.5 & 87.5 & 13.6 & 91.9 \\
\hline 8 & 98.3 & 80.3 & 27.4 & 134.1 \\
\hline
\end{tabular}

The success rate refers to the extraction of the MHR from the raw data.

${ }^{\mathrm{a} M H R}=$ Maternal heart rate

${ }^{\mathrm{b}} \mathrm{STV}=$ short term variability

${ }^{c} \mathrm{MMR}=$ Mean minute range

\section{Discussion}

\section{Baseline variability}

Heart rate variability (HRV) is the variation in HR over time and is due to variation in rapid (beat to beat), slow (seconds to minutes), and transient, event-related, changes in HR. It is predominantly dependent on extrinsic regulation of heart rate [13]. Assessment of HRV as a marker of ANS function and the adaptations to different physiological and pathological conditions is now receiving more attention [4]. HRV may be affected by different physiological and pathological conditions such as age, gender, fatigue, emotions, drugs, smoking, alcohol consumption, myocardial infection, arterial hypertension, central nervous system disorders and renal failure. In addition, mood and anxiety disorders are associated with abnormal ANS function and cause a reduction in HRV [14].

Rouleau et al. [15] examined whether decreased high-frequency (HF) variability mediated the association between depressed mood and gestational hypertension in 287 pregnant women. They found that depressive symptoms were associated with less HF HRV and that there was an indirect effect of depressed mood on gestational hypertension HF HRV. They concluded that cardiac vagal control was a possible pathway through which prenatal depressed mood was associated with gestational hypertension.

Murphy et al. [16] studied the effect of pregnancy on postpartum parameters of the electrocardiogram, and determined how a history of pre-eclampsia affected these parameters. Participants consisted of non-pregnant controls, normotensive parous controls and women with a recent history of pre-eclampsia. The reductions in HRV, induced by uncomplicated pregnancy, returned to non-pregnant levels 6-8 months after delivery, but in pre-eclamptic postpartum women the HRV remained reduced compared to the controls.

In a case control study, Braeken et al. [17] examined whether anxiety-related conditions, such as reduced autonomic cardiac control, reflected by reduced HRV, could persist after correction of the disorder. They found that pregnant women with previous but not current anxiety and their children had lower HRV. A model of autonomic imbalance may provide a unifying framework within which to investigate the impact of risk factors, including psychosocial factors and work stress, on cardiovascular disease [18].

\section{Basal heart rate}

Tachycardia: The maternal heart rate begins to increases during the first few weeks of pregnancy and peaks in the late second to early third trimester [19]. After 32 weeks, the maintenance of cardiac output becomes more dependent on heart rate due to the fall in stroke volume. During pregnancy, the mean maternal heart rate usually increases by an average of 10 to 20 beats per minute [19]. Tachycardia is therefore defined as a rate of at least 100 beats per minute under resting conditions [20].

Few articles in the literature address maternal tachycardia but it is referred to when cardiac disease or the use of sympathomimetics are investigated. Nel examined 1431 participants of the Safe Passage Study. Tachycardia was found in $7.1 \%$ of these cases. When they were compared to controls at similar gestations without tachycardia, no unexpected medical conditions or poor perinatal outcome were detected. Interestingly, the birth weights in the tachycardia group 
were significantly higher and the difference could not be explained by cigarette smoking, gestational age or sex of the newborn [21]. However, Carson et al. [22] found a higher prevalence of tachycardia (29\%) in healthy, asymptomatic patients in the third trimester of pregnancy.

Bradycardia: The definition of bradycardia in pregnancy has not been standardized. Vasapollo et al. [23] compared MHRs in primigravidae with intrauterine growth restriction to normal control pregnancies and found the pulse rates were $82 \mathrm{bpm}$ and $86 \mathrm{bpm}$ $(\mathrm{p}=0.03)$ respectively. Everette et al. [24] studied 99 pregnant women between 23 and 30 weeks. They found a significant correlation between MHR and birth weight $\mathrm{z}$-scores $(\mathrm{r}=0.22, \mathrm{p}=0.03)$ and suggested that a reduced maternal cardiac output with a lower MHR may indicate poor cardiovascular adaptation in early pregnancy.

\section{Periodic changes}

To determine the effect of maternal position on periodic changes of the maternal heart rate Ibrahim $e t$ al. [25] did a prospective observational study in pregnant women before elective caesarean section. Continuous transabdominal non-invasive recording of $\mathrm{MHR}$, fetal heart rate patterns and uterine activity were done for one hour in 119 women. Maternal position was changed every 15 minutes from lateral, to supine, then the other lateral and then supine again. MHRs were four beats per minute slower in the left lateral position when compared to the right lateral position. Periodic maternal heart rate changes were seen in $10.9 \%$ of participants. Most of these (84.6\%) were associated with uterine activity and not maternal position. They concluded that in a subgroup of pregnant women at term, uterine activity was associated with periodic changes of MHR. In low risk pregnancies there seemed to be no effect on the fetal heart rate pattern but the implications for the compromised fetus are still unsure. They postulated that displacement of blood from the uterus and choriodecidual space into the venous circulation increases the preload to the heart which then increases myocardial contractility and is associated with a short increase in MHR [3].

They also stated that mechanism for periodic decline of the MHR is different, as aortocaval compression during the supine position could play a role as it could lead to decreased venous return to the heart and this subsequent decrease in preload could cause deceleration of the MHR. They suspected that reduced venous return to the heart could still occur in the lateral positions as they also found a decline in the MHR in these positions. An explanation for this was that aortacaval compression probably occurs more often when the uterus contracts. When the uterus is relaxed it would mold around the great vessels, causing little compression. However, during contraction, compression could happen more readily, even on the lateral side as the firmer uterus would then simultaneously compress the inferior cava and anterior abdominal wall.

We have observed many unusual MHR patterns, but the clinical significance of these patterns is unclear. We are pursuing this issue through analyses of the recordings of more than 6,000 participants in the Safe Passage Study and linking the quantification of these patterns to reviews of the pregnancy outcomes to determine possible associations between the different maternal heart rate patterns and maternal and fetal outcome.

\section{Ethics approval}

The SPS was approved by the Health Research Ethics Committee of Stellenbosch University (approval number N06/10/210).

\section{Author contributions}

Prof Odendaal is the local principal investigator of the Safe Passage Study. He initiated this study, selected the various maternal heart rate patterns, wrote the initial draft and coordinated the editing.

Dr Groenewald is the manager of the Safe Passage Study. He supervised all activities and contributed to the writing and editing of the manuscript.

Dr Fifer and Dr Myers are both members of the PASS Network. They initiated all the physiological assessments, supervised the quality control and participated in the writing and editing the manuscript.

\section{Acknowledgements}

The study was funded by the National Institute on Alcohol Abuse and Alcoholism, Eunice Kennedy Shriver National Institute of Child Health and Human Development, and National Institute on Deafness and Other Communication Disorders: U01 HD055154, U01 HD045935, U01 HD055155, U01 HD045991, and U01 AA016501.

The manuscript was approved by the NICHD.

\section{Informed consent}

Written informed consent was obtained from all participants and where possible also the father of the infant. A copy of the signed consent form, in their home language, was given to the mother or parents to keep.

\section{Conflicts of interest}

The authors have no conflict of interest to declare.

\section{References}

1. Gelson E, Curry R, Gatzoulis MA, Swan L, Lupton M, et al. (2011) Effect of maternal heart disease on fetal growth. Obstet Gynecol 117: 886-891. [Crossref]

2. Herrey A, Nelson-Piercy C (2010) Cardiovascular disease in pregnancy. Medicine 38 555-560.

3. Kobirumaki-Shimozawa F, Inoue T, Shintani SA, Oyama K, Terui T, et al. (2014) Cardiac thin filament regulation and the Frank-Starling mechanism. J Physiol Sci 64: 221-232. [Crossref]

4. Taralov ZZ, Terziyski KV, Kostianev SS (2015) Heart rate variability as a method for assessment of the autonomic nervous system and the adaptations to different physiological and pathological conditions. Folia Med (Plovdiv) 57: 173-180. [Crossref]

5. Odendaal HJ (1976) False interpretation of fetal heart role monitoring in cases of intrauterine death. S Afr Med J 50: 1963-1965. [Crossref]

6. Sherer DM, Dalloul M, Pierre N, Abulafia O (2005) Intrapartum repetitive maternal heart rate deceleration pattern simulating nonreassuring fetal status. Am J Perinatol 22: 165-167. [Crossref]

7. Bakker PC, Colenbrander GJ, Verstraeten AA, Van Geijn HP (2004) The quality of intrapartum fetal heart rate monitoring. Eur J Obstet Gynecol Reprod Biol 116: 22-27. [Crossref]

8. Muñoz Brands RM, Bakker PC, Bolte AC, van Geijn HP (2009) Misidentification of maternal for fetal heart rate patterns after delivery of the first twin. J Perinat Med 37: 177-179. [Crossref]

9. Reinhard J, Hayes-Gill BR, Schiermeier S, Hatzmann W, Herrmann E, et al. (2012) Intrapartum signal quality with external fetal heart rate monitoring: A two way trial of external Doppler CTG ultrasound and the abdominal fetal electrocardiogram. Arch Gynecol Obstet. [Crossref]

10. Vasak B, Graatsma EM, Hekman-Drost E, Eijkemans MJ, Schagen Van Leeuwen JH, et al. (2013) Uterine electromyography for identification of first-stage labor arrest in term nulliparous women with spontaneous onset of labor. Am J Obstet Gynecol. [Crossref]

11. Dukes KA, Burd L, Elliott AJ, Fifer WP, Folkerth RD, et al. (2014) The safe passage study: Design, methods, recruitment, and follow-up approach. Paediatr Perinat Epidemiol 28: 455-465. [Crossref] 
12. Hofmeyr F, Groenewald CA, Nel DG, Myers MM, Fifer WP, et al. (2014) Fetal heart rate patterns at 20 to 24 weeks gestation as recorded by fetal electrocardiography. $J$ Matern-Fetal Neonatal Med 27: 714-718. [Crossref]

13. Rajendra Acharya U, Paul Joseph K, Kannathal N, Lim CM, Suri JS (2006) Heart rate variability: a review. Med Biol Eng Comput 44: 1031-1051. [Crossref]

14. Kemp AH, Quintana DS, Gray MA, Felmingham KL, Brown K, et al. (2010) Impact of depression and antidepressant treatment on heart rate variability: a review and metaanalysis. Biol Psychiatry 67: 1067-1074. [Crossref]

15. Rouleau CR, Tomfohr-Madsen LM, Campbell TS, Letourneau N, O'Beirne M, et al (2016) The role of maternal cardiac vagal control in the association between depressive symptoms and gestational hypertension. Biol Psychol 117: 32-42. [Crossref]

16. Murphy MS, Seaborn GE, Redfearn DP, Smith GN (2015) Reduced Heart Rate Variability and Altered Cardiac Conduction after Pre-Eclampsia. PLoS One 10: e0138664. [Crossref]

17. Braeken MAKA, Kemp AH, Outhred T, Otte RA, Monsieur GJYJ, et al. (2013) Pregnant mothers with resolved anxiety disorders and their offspring have reduced heart rate variability: Implications for the health of children. PLOS ONE 8.

18. Thayer JF, Yamamoto SS, Brosschot JF (2010) The relationship of autonomic imbalance, heart rate variability and cardiovascular disease risk factors. Int J Cardiol 141: 122-131. [Crossref]
19. Easterling TR, Benedetti TJ, Schmucker BC, Millard SP (1990) Maternal hemodynamics in normal and preeclamptic pregnancies: a longitudinal study. Obstet Gynecol 76: 1061-1069. [Crossref]

20. Klein LL, Galan HL (2004) Cardiac disease in pregnancy. Obstet Gynecol Clin North Am 31: 429-459. [Crossref]

21. Nel N (2012) Prevalence of maternal tachycardia during late pregnancy. MCur theses, Stellenbosch University, 2012

22. Carson MP, Powrie RO, Rosene-Montella K (2002) The effect of obesity and position on heart rate in pregnancy. J Matern Fetal Neonatal Med 11: 40-45. [Crossref]

23. Vasapollo B, Valensise H, Novelli GP, Larciprete G, Di Pierro G, et al. (2002) Abnormal maternal cardiac function and morphology in pregnancies complicated by intrauterine fetal growth restriction. Ultrasound Obstet Gynecol 20: 452-457. [Crossref]

24. Everett TR, Mahendru AA, McEniery CM, Wilkinson IB, Lees CC (2013) Midtrimester maternal heart rate is related to neonatal birth weight. J Matern Fetal Neonatal Med 26: 1082-1085.

25. Ibrahim S, Jarefors E, Nel DG, et al. (2015) Effect of maternal position and uterine activity on periodic maternal heart rate changes before elective cesarean section at term. Acta Obstet Gynecol Scand 94: 1359-1366. [Crossref]

Copyright: $\odot 2018$ Odendaal H. This is an open-access article distributed under the terms of the Creative Commons Attribution License, which permits unrestricted use, distribution, and reproduction in any medium, provided the original author and source are credited. 